\title{
Detection of alphacoronavirus in bat fecal samples from Volgograd region
}

\author{
E.V. Korneenko \\ Central Research Institute of \\ Epidemiology, Moscow, Russia, \\ lennatta@yandex.ru \\ A.V. Dudorova \\ Lomonosov Moscow State University, \\ Moscow, Russia \\ E.V. Pimkina \\ Central Research Institute of \\ Epidemiology, Moscow, Russia
}

\author{
A.E. Samoilov \\ Central Research Institute of \\ Epidemiology, Moscow, Russia \\ andrei.samoilov@gmail.com \\ V.G. Dedkov \\ Pasteur Institute, Saint-Petersburg, \\ Saint-Petersburg, Russia \\ M.V. Safonova \\ Plague Control Center, Federal Service \\ on Consumers' Rights Protection and \\ Human Well-Being Surveillance, \\ Moscow, Russia
}

\author{
I.V.Artyushin \\ Lomonosov Moscow State University, \\ Moscow, Russia, sometyx@gmail.com \\ A.D. Matsvay \\ FSBI "Center of Strategic Planning" of \\ the Ministry of Health, Moscow, Russia \\ A.S. Speranskaya \\ Central Research Institute of \\ Epidemiology, Moscow, Russia, \\ Lomonosov Moscow State University, \\ Moscow Russia, \\ hanna.s.939@gmail.com
}

\begin{abstract}
Bats are natural reservoirs of many emerging viruses and their virome can differ according to the regions. In this study we have found a genome fragment of Coronaviridae which was $99 \%$ percent identical to Alphacoronavirus N.las/C/Spain/2007 in bat fecal sample from Volgograd region

\footnotetext{
Keywords - bats, viruses, coronavirus, high throughput
} sequencing
\end{abstract}

\section{Motivation and aim}

Bats are natural reservoirs of many emerging viruses and their virome can differ according to the regions. The aim of this study was to analyse virome of bat fecal samples which were collected in Volgograd region (Russian Federation).

\section{Materials and methods}

Fecal samples from bat species (Nyctalus noctula, Vespertilio murinus, Nyctalus lesleri, Myotis daubentonii, Eptesicus serotinus, Pipistrellus kuhlii) were collected in 2016 in Volgograd regions (Yaminsky farm, Zimnyatskiy farm) and transported to laboratory of Moscow State University (MSU) in RNAlater solution. Notably, that transportation took some days and no special conditions were applied. Then, extraction using RNeasy Lipid Tissue Mini Kit (Qiaqen, Germany), reverse transcription using REVERTA-L (AmpliSens, Russia), PCR amplification on primers described in previous study [1] and library preparation was performed using Illumina TruSeq (in house) protocol. Libraries were sequenced using Illumina MiSeq platform. High throughput sequencing data were assessed with FastQC, filtered by trimming using trimmomatic 0.38 , assembled by SPAdes 3.13 , taxonomy of resulting contigs was identified by the BLASTn.

\section{Results and discussion}

For 26 samples we performed library preparation and sequencing on Illumina Miseq Platform. In one sample we have found a genome fragment of Coronaviridae which was 99\% percent identical to Alphacoronavirus N.las/C/Spain/2007 accession number -HQ184051.1. Interestingly, this virus was found in a greater noctule bat (Nyctalus lasiopterus) in Spain in 2007 [2]. According to taxonomy Alphacoronavirus N.las/C/Spain/2007 does not relates to the aetiological agents of severe acute respiratory syndrome (SARS), which was identified as a $2 \mathrm{~b} \beta-\mathrm{CoV}$, Middle East respiratory syndrome-CoV (MERS-CoV) is as 2c $\beta-\mathrm{CoV}$ or coronavirus disease 2019 (COVID-19) SARS-CoV2. This finding can present interest for the further study of virus migration in bats in different regions of Russia Federation.

\section{REFERENCES}

[1] Virome analysis for identification of viruses in bat species from Moscow region, Speranskaya A.S., Pimkina E.V., Artyushin I.V., Shipulin G.A., Deviatkin A.A., Kuleshov K.V., Dedkov V.G., Safonova M.V., Bioinformatics of Genome Regulation and Structure/BGRS-2016

[2] Mol. Biol. Evol., 28 (2011), pp. 2731-2739

[3] Lelli, D.; Papetti, A.; Sabelli, C.; Rosti, E.; Moreno, A.; Boniotti, M.B. Detection of Coronaviruses in Bats of Various Species in Italy.Viruses 2013, 5, 2679-2689 